tion in the sgk1-knockout mouse. J. Clin. Invest. 110:1263-1268. doi:10.1172/JCI200215696.

9. Bhalla, V., Soundararajan, R., Pao, A.C., Li, H., and Pearce, D. 2006. Disinhibitory pathways for control of sodium transport: regulation of $\mathrm{ENaC}$ by SGK1 and GILZ. Am. J. Physiol. Renal Physiol. 291:F714-F721.

10. Zhang, W., et al. 2007. Aldosterone-induced Sgk1 relieves Dot1a-AF9-mediated transcriptional repression of epithelial $\mathrm{Na}^{+}$channel $\alpha$. J. Clin. Invest. 117:773-783. doi:10.1172/JCI29850.

11. Mick, V.E., et al. 2001. The alpha-subunit of the epithelial sodium channel is an aldosterone-induced transcript in mammalian collecting ducts, and this transcriptional response is mediated via distinct cis-elements in the 5 -flanking region of the gene. Mol. Endocrinol. 15:575-588.

12. Lee, D.Y., Teyssier, C., Strahl, B.D., and Stallcup, M.R. 2005. Role of protein methylation in regulation of transcription. Endocr. Rev. 26:147-170.

13. Feng, Q., et al. 2002. Methylation of H3-lysine 79 is mediated by a new family of HMTases without a SET domain. Curr. Biol. 12:1052-1058.

14. van Leeuwen, F., Gafken, P.R., and Gottschling, D.E. 2002. Dot $1 p$ modulates silencing in yeast by methylation of the nucleosome core. Cell. 109:745-756.
15. Ng, H.H., et al. 2002. Lysine methylation within the globular domain of histone $\mathrm{H} 3$ by Dot 1 is important for telomeric silencing and Sir protein association. Genes Dev. 16:1518-1527.

16. Zhang, W., et al. 2006. Aldosterone-sensitive repression of ENaCalpha transcription by a histone $\mathrm{H} 3$ lysine-79 methyltransferase. Am. J. Physiol., Cell Physiol. 290:C936-C946.

17. Zhang, W., Xia, X., Reisenauer, M.R., Hemenway, C.S., and Kone, B.C. 2006. Dot1a-AF9 complex mediates histone $\mathrm{H} 3$ Lys-79 hypermethylation and repression of ENaCalpha in an aldosterone-sensitive manner. J. Biol. Chem. 281:18059-18068.

\title{
HDL proteomics: pot of gold or Pandora's box?
}

\author{
Muredach P. Reilly ${ }^{1}$ and Alan R. Tall ${ }^{2}$ \\ ${ }^{1}$ Cardiovascular Institute, Department of Medicine, University of Pennsylvania Medical Center, Philadelphia, Pennsylvania, USA. \\ ${ }^{2}$ Division of Molecular Medicine, Department of Medicine, Columbia University Medical Center, New York, New York, USA.
}

\begin{abstract}
In this issue of the JCI, Vaisar et al. studied the proteome of HDL (see the related article beginning on page 746 ). They reveal, quite unexpectedly, that HDL is enriched in several proteins involved in the complement cascade, as well as in a variety of protease inhibitors, supporting the concept that HDL plays a role in innate immunity and in the regulation of proteolytic cascades involved in inflammatory and coagulation processes. The protein makeup of HDL also appears to be altered in patients with coronary artery disease. HDL proteomics is in its infancy, and preliminary findings will need to be confirmed using standardized approaches in larger clinical samples. However, this approach promises to better elucidate the relationship of HDL to atherosclerosis and its complications and could eventually help in the development of biomarkers to predict the outcome of interventions that alter HDL levels and functions.
\end{abstract}

The inverse relationship between plasma HDL-cholesterol (HDL-C) levels and atherosclerotic cardiovascular disease (CVD) provides the epidemiological basis for the widely accepted hypothesis that HDL is atheroprotective. Despite intense research, the underlying mechanisms of HDL atheroprotection remain incompletely understood. Indeed, recent clinical trials $(1,2)$ indicate the complexity of HDL physiology and the challenges in developing HDL therapies. HDL function, and benefit with a specific therapy, may depend more on

Nonstandard abbreviations used: $\mathrm{ABCA} 1, \mathrm{ABC}$ transporter $\mathrm{A} 1$; CAD, coronary artery disease; $\mathrm{CE}$, cholesterol ester; CETP, CE transfer protein; CVD, cardiovascular disease; HDL-C, HDL-cholesterol; ILLUMINATE, Investigation of Lipid Level Management to $\underline{\text { Understand Its }}$ Impact in ATherosclerotic Events; LCAT, lecithin:cholesterol acyltransferase; Lp-PLA 2 , lipoprotein-associated phospholipase $\mathrm{A}_{2}$; RCT, reverse cholesterol transport; SR-BI, scavenger receptor BI.

Conflict of interest: Muredach P. Reilly is in receipt of research funding from GlaxoSmithKline. Alan R. Tall has acted as a consultant to Bristol-Myers Squibb, Merck, Pfizer, Reddy Pharma, and Wyeth.

Citation for this article: J. Clin. Invest. 117:595-598 (2007). doi:10.1172/JCI31608. the molecular mechanism driving increases in HDL-C than on the absolute level of HDL-C (3). Some interventions that raise HDL-C levels may have no benefit and even promote atherosclerosis (4), while other therapies may reduce CVD without actually changing HDL-C levels (1). Overall, the epidemiological evidence suggests that the majority of mechanisms that result in higher HDL-C levels in vivo will provide atheroprotection; the question is how to identify such targets. This requires a shift in mindset toward assessing HDL in terms of its atheroprotective functions rather than just levels of cholesterol and its main apoprotein, APOAI.

\section{Proposed mechanisms of HDL atheroprotection}

Experimental studies, including limited work in humans, suggest several distinct but potentially overlapping HDL atheroprotective functions. These include reverse cholesterol transport (RCT) (5) and reductions in oxidative stress and in innate immune inflammation $(6,7)$.
RCT. The ability of HDL to promote RCT has been thought of as the major function of HDL for more than four decades $(8,9)$, although convincing demonstration of this process in vivo has only emerged in the past few years (5). In atherosclerosis the primary cell that is loaded with cholesterol is the arterial macrophage - therefore, it may make more sense to conceptualize RCT in terms of macrophage cholesterol efflux potential or "macrophage RCT" rather than in terms of total peripheral tissue cholesterol RCT $(5,9)$.

The first step in macrophage RCT is efflux of cholesterol from arterial macrophages, a highly regulated process involving specific transporters including $A B C$ transporter $\mathrm{A} 1$ (ABCA1) and ABCG1 (5). ABCA1 facilitates efflux of cholesterol and phospholipids (including oxidized phospholipids) to lipid-poor APOAI, whereas ABCG1-mediated cholesterol efflux to more mature HDL particles (10) is enhanced by lecithin:cholesterol acyltransferase (LCAT) and APOE in HDL (11) and may be in part be responsible for the "passive cholesterol efflux" characterized by Rothblat, Phillips, and coworkers (12). Expression of both transporters is upregulated in macrophages by oxysterols that activate the nuclear hormone receptor liver X receptors (LXRs) and directly target the promoters of theses genes. Macrophage expression of both ABCA1 and ABCG1 enhances macrophage cholesterol efflux and protects against experimental atherosclerosis.

Following efflux to HDL and esterification by LCAT, transport of cholesterol to the liver is mediated directly by HDL hepatic receptors, including scavenger 
Table 1

Assays of HDL function in humans

\section{Assay class \\ HDL subpopulations and size \\ Assay \\ 2D PAGE (27)}

NMR (28)

RCT

\author{
Macrophage cholesterol \\ efflux (12)
}

Fecal sterol excretion (29)

HDL tracer kinetic studies (30)

Activity and mass assays of CEPT, LCAT, lipases etc.

HDL antiinflammatory

Monocyte chemotactic assay $(7,31)$; cell-free assay $(7,31)$

Vascular adhesion molecular expression or levels

HDL antioxidant $\mathrm{Lp}-\mathrm{PLA} \mathrm{A}_{2}$ mass or activity

\author{
Advantages/limitations \\ Identifies particles that may relate to HDL \\ function and outcomes; low throughput, \\ semiquantitative, surrogate of true function \\ Identifies HDL particle size and number; \\ high throughput, but limited evidence \\ for CVD risk prediction beyond HDL-C \\ Analyzes ex vivo capacity of isolated \\ HDL to efflux cholesterol from \\ macrophages; low throughput \\ Estimates total body excretion \\ of cholesterol; may lack sensitivity for \\ macrophage RCT and be confounded \\ by bowel cholesterol metabolism \\ Trace HDL lipid fluxes and excretion \\ from body; do not assay macrophage \\ $\mathrm{RCT}$; hepatic and bowel activity \\ confounds tracer kinetics \\ Estimate mass or activity of \\ HDL proteins involved in RCT; \\ activity assays require standardization \\ Analyzes ex vivo capacity of HDL \\ to suppress LDL-induced chemotaxis; \\ low throughput, lacks standardization \\ Requires vascular tissue or plasma; plasma \\ assays are not specific to HDL function \\ Assays HDL antioxidant enzymes; \\ single dimension of HDL function, \\ lacks standardization
}

\author{
Human studies \\ Yes, small- to intermediate- \\ sized studies \\ Yes, epidemiological and \\ clinical trials \\ Yes, small scale; relationship \\ to atherosclerosis is \\ lacking \\ Yes, small scale; relationship \\ to atherosclerosis is \\ lacking \\ Yes, proof of concept; \\ needs validation \\ Yes; greater evidence of \\ relationship to RCT and \\ atherosclerosis is required \\ Yes, proof-of concept studies
}

Yes, plasma assays

Yes, limited proof of concept

$\mathrm{RCT}$, reverse cholesterol transport.

receptor BI (SR-BI), or indirectly by cholesterol ester (CE) transfer protein-driven (CETP-driven) CE transfer to apoB lipoproteins and liver uptake (5). Hepatic SR-BI mediates selective uptake of HDL-CE and free cholesterol without concomitant uptake of HDL protein (4). Despite reducing plasma HDL-C levels, hepatic SR-BI overexpression in mice enhances macrophage RCT and reduces atherosclerosis $(4,5)$. The role of SR-B1 in human physiology remains uncertain, however, because relatively little HDL-CE is taken up directly by the liver in humans (13).

CETP mediates the exchange of HDL-CE for triglyceride on apoB lipoproteins. CETPdeficient humans have extremely high HDL-C levels and slow turnover of APOAI; however, the role of CETP in RCT and atherosclerosis remains uncertain $(3,5,14)$. Pharmacological inhibition of CETP results in increased levels of large HDL particles (15). It is possible that inhibition of CETP results in a switch from an ABCA1- to an ABCG1-mediated cholesterol efflux pathway $(10,11)$. At present, it is unclear what might be the optimal level of CETP inhibition for increasing macrophage cholesterol efflux. Also, the failure of the CETP inhibitor torcetrapib in the ILLUMINATE (Investigation of Lipid Level Management to Understand Its Impact in ATherosclerotic Events) trial likely involved non-HDL-related toxicity, such as off-target hypertensive side effects (2). It will be interesting to see whether changes in HDL levels, subclasses, or function were predictive of the outcome in the ILLUMINATE study or whether CETP inhibition promotes or retards novel measures of RCT in experimental models. However, the failure of torcetrapib in the ILLUMINATE study dramatically illustrates the need for plasma or other surrogate biomarkers that faithfully reflect the underlying antiatherogenic properties of HDL.

Antiinflammatory and antioxidant effects of HDL. A body of literature has emerged supporting specific antiinflammatory and antioxidant effects of $\operatorname{HDL}(6,7)$. Remarkably, in this issue of the JCI, Vaisar and colleagues (16), using a proteomics approach, found that more HDL proteins are involved in immune/inflammatory functions (23 of
48 proteins) than in lipid transport and metabolism (22 proteins), suggesting a fundamental role for HDL in innate immunity. Indeed, HDL binds to and modulates the actions of endotoxin and other bacterial antigens, provides a platform for assembly of innate immune complexes, acts as an acceptor for oxidized phospholipids, and blocks oxidation of apoB lipoproteins $(6,7,17,18)$. HDL levels fall during acute inflammation, perhaps to achieve conditions permissive for acute inflammation. Two independent proteomic analyses have revealed HDL enrichment in proteins regulating complement, proteolysis, and coagulation $(16,19)$, suggesting modulation of inflammation-induced tissue injury and hemostasis. The recovery of HDL levels following the acute inflammatory response could play an important role in suppressing ongoing inflammation.

Modifications of HDL that occur during the acute-phase response are similar to those observed chronically in atherosclerosis $(6,7)$. A number of HDL-associated antioxidant enzymes, including paraoxonase and lipoprotein-associated phospho- 
lipase $A_{2}\left(L p-P L A_{2}\right)$, promote catabolism of oxidized phospholipids. Accumulation of oxidized lipids, however, negatively regulates the activities of these enzymes. Using cell-based assays of monocyte chemotactic activity or cell-free assays of oxidation, Fogelman and colleagues have shown that HDL particles isolated during the acute phase and from patients with coronary artery disease (CAD) fail to retard, and in fact enhance, LDL-mediated inflammation (7). Notably, a convergence of HDL antiinflammatory functions with its RCT functions has emerged - HDL isolated from CAD patients contains a specific myeloperoxidase-driven tyrosine modification of APOAI that coincides with attenuation of cholesterol efflux via $\operatorname{ABCA1}(20,21)$.

\section{Measures of HDL atheroprotective function and the HDL proteome}

We continue to have limited insight into the precise mechanisms of HDL atheroprotection, in part, due to our inability to assess HDL functions in vivo. Although several measures of HDL particles, composition, and function exist (Table 1), there are no tractable methods for assessing RCT in humans, and simple, reliable, and reproducible assays of HDL antiinflammatory functions are lacking. In this setting, recent HDL proteomic studies may provide novel insights into HDL physiology and the potential for development of bioassays of HDL function, although such studies are in their infancy $(16,19,22-24)$.

In this issue, Vaisar et al. present the largest and most comprehensive mass spectrometry-based study to date of the HDL proteome (16). Arguably, the most striking aspect of these early studies is not just the diversity of the HDL proteins and peptides $(16,19)$ identified but also the overrepresentation of proteins involved in several non-lipid transport functions, including the acutephase response, complement regulation, proteolysis, and coagulation $(16,19,22,24)$ (Supplemental Table 1; supplemental material available online with this article; doi:10.1172/JCI31608DS1), suggesting novel HDL functions. Although convincing evidence of functional roles for HDL in these processes is limited, past and emerging studies have shown that HDL and/or APOAI can attenuate response to experimental endotoxemia (17), inhibit complement activation (25), and inhibit platelet activation, serpins, and thrombosis (26).

The current study (16) is notable for examining a large human sample (albeit including only 33 subjects), comparing HDL proteins isolated from the plasma of healthy controls with HDL proteins from patients with CAD $(n=7)$ and with HDL isolated from human atherosclerotic plaques. Importantly, semiquantitative peptide counting linked to appropriate statistical methodologies was applied to assess relative protein abundance and provided evidence that HDL in CAD is enriched in APOE and complement components 3 and 4 , protein changes that may relate to both RCT and antiinflammatory functions. The observation that the plasma HDL in CAD contains a subset of proteins found on HDL in atherosclerotic plaques requires validation but implies that the HDL proteome can provide a window into plaque activity. Whether such protein changes, and which ones, are measures of HDL atheroprotective functions has yet to be determined.

The degree of variation across studies in the number and identity of proteins associated with HDL (Supplemental Table 1; refs. $16,19,22-24)$ raises some concerns. This is likely related to technical differences in HDL isolation and subsequent methodology and highlights the need for technical standardization and rigorous external validation.

\section{Future directions}

In moving forward, the challenge will be to develop relatively simple HDL biomarkers that can be measured before and after a clinical intervention, then correlated with clinical outcomes or atherosclerosis imaging, and ultimately used in CVD risk prediction. Examples could be an immunoassay for the content of specific proteins in HDL or its subfractions; e.g., the proportion of HDL or subclasses containing APOE, LCAT, or Lp-PLA 2 , specific complement proteins, antiproteases, or oxidized lipids could turn out to have predictive value. While more complicated measurements, such as macrophage RCT or even cell-based assays of macrophage cholesterol efflux using HDL isolated before and after intervention, are less likely to be applicable on a large scale or validated against clinical outcomes, they may be critical for proof of concept of novel therapies and understanding the functional properties of simpler HDL biomarkers. Application of a broad spectrum of assays that address HDL functionality as well as composition is likely to provide the greatest insight into the relationship between HDL and atherosclerosis and the effects of novel therapies. Indeed, failure to apply such measures as a complement to atherosclerosis imaging will reduce the likelihood of developing HDL-related prognostic and therapeutic strategies.

Address correspondence to: Muredach P. Reilly, Cardiovascular Institute, University of Pennsylvania Medical Center, 909 BRB 2/3, 421 Curie Blvd., Philadelphia, Pennsylvania 19104-6160, USA. Phone: (215) 573-1214; Fax: (215) 573-2094; E-mail: muredach@ itmat.gcrc.upenn.edu.

1. Nissen, S.E., et al. 2003. Effect of recombinant ApoA-I Milano on coronary atherosclerosis in patients with acute coronary syndromes: a randomized controlled trial. JAMA. 290:2292-2300.

2. FDA. 2006. Pfizer stops all torcetrapib clinical trials in interest of patient safety [press release]. http://www.fda.gov/bbs/topics/NEWS/2006/ NEW01514.html.

3. Cuchel, M., and Rader, D.J. 2003. Genetics of increased HDL cholesterol levels: insights into the relationship between HDL metabolism and atherosclerosis. Arterioscler. Thromb. Vasc. Biol. 23:1710-1712.

4. Trigatti, B.L. 2005. Hepatic high-density lipoprotein receptors: roles in lipoprotein metabolism and potential for therapeutic modulation. Curr. Atheroscler. Rep. 7:344-350.

5. Cuchel, M., and Rader, D.J. 2006. Macrophage reverse cholesterol transport: key to the regression of atherosclerosis? Circulation. 113:2548-2555.

6. Barter, P.J., et al. 2004. Antiinflammatory properties of HDL. Circ. Res. 95:764-772.

7. Navab, M., et al. 2006. Mechanisms of disease: proatherogenic HDL - an evolving field. Nat. Clin. Pract. Endocrinol. Metab. 2:504-511.

8. Glomset, J.A. 1980. High-density lipoproteins in human health and disease. Adv. Intern. Med. 25:91-116.

9. Tall, A.R., Wang, N., and Mucksavage, P. 2001. Is it time to modify the reverse cholesterol transport model? J. Clin. Invest. 108:1273-1275. doi:10.1172/ JCI200114342.

10. Wang, N., Ranalletta, M., Matsuura, F., Peng, F., and Tall, A.R. 2006. LXR-induced redistribution of ABCG1 to plasma membrane in macrophages enhances cholesterol mass efflux to HDL. Arterioscler. Thromb. Vasc. Biol. 26:1310-1316.

11. Matsuura, F., Wang, N., Chen, W., Jiang, X.C., and Tall, A.R. 2006. HDL from CETP-deficient subjects shows enhanced ability to promote cholesterol efflux from macrophages in an apoE- and ABCG1dependent pathway. J. Clin. Invest. 116:1435-1442. doi:10.1172/JCI27602.

12. Yancey, P.G., et al. 2003. Importance of different pathways of cellular cholesterol efflux. Arterioscler. Thromb. Vasc. Biol. 23:712-719.

13. Schwartz, C.C., VandenBroek, J.M., and Cooper, P.S. 2004. Lipoprotein cholesteryl ester production, transfer, and output in vivo in humans. J. Lipid Res. 45:1594-1607.

14. Curb, J.D., et al. 2004. A prospective study of HDL$\mathrm{C}$ and cholesteryl ester transfer protein gene mutations and the risk of coronary heart disease in the elderly. J. Lipid Res. 45:948-953.

15. Brousseau, M.E., et al. 2004. Effects of an inhibitor of cholesteryl ester transfer protein on HDL cholesterol. N. Engl. J. Med. 350:1505-1515.

16. Vaisar, T., et al. 2007. Shotgun proteomics implicates protease inhibition and complement activation in the antiinflammatory properties of HDL. J. Clin. Invest. 117:746-756. doi:10.1172/JCI26206.

17. Wu, A., Hinds, C.J., and Thiemermann, C. 2004. 
High-density lipoproteins in sepsis and septic shock: metabolism, actions, and therapeutic applications. Shock. 21:210-221.

18. Shiflett, A.M., Bishop, J.R., Pahwa, A., and Hajduk, S.L. 2005. Human high density lipoproteins are platforms for the assembly of multi-component innate immune complexes. J. Biol. Chem. 280:32578-32585

19. Rezaee, F., Casetta, B., Levels, J.H., Speijer, D., and Meijers, J.C. 2006. Proteomic analysis of high-density lipoprotein. Proteomics. 6:721-730.

20. Zheng, L., et al. 2005. Localization of nitration and chlorination sites on apolipoprotein A-I catalyzed by myeloperoxidase in human atheroma and associated oxidative impairment in ABCA1-dependent cholesterol efflux from macrophages. J. Biol. Chem. 280:38-47.

21. Shao, B., et al. 2006. Myeloperoxidase impairs ABCA1-dependent cholesterol efflux through methionine oxidation and site-specific tyrosine chlorination of apolipoprotein A-I. J. Biol. Chem. 281:9001-9004.
22. Heller, M., et al. 2005. Mass spectrometry-based analytical tools for the molecular protein characterization of human plasma lipoproteins. Proteomics. 5:2619-2630.

23. Karlsson, H., Leanderson, P., Tagesson, C., and Lindahl, M. 2005. Lipoproteomics II: mapping of proteins in high-density lipoprotein using two-dimensional gel electrophoresis and mass spectrometry. Proteomics. 5:1431-1445.

24. Hortin, G.L., Shen, R.F., Martin, B.M., and Remaley, A.T. 2006. Diverse range of small peptides associated with high-density lipoprotein. Biochem. Biophys. Res. Commun. 340:909-915.

25. Hamilton, K.K., Zhao, J., and Sims, P.J. 1993. Interaction between apolipoproteins A-I and A-II and the membrane attack complex of complement. Affinity of the apoproteins for polymeric C9.J. Biol. Chem. 268:3632-3638.

26. Mineo, C., Deguchi, H., Griffin, J.H., and Shaul, P.W. 2006. Endothelial and antithrombotic actions of HDL. Circ. Res. 98:1352-1364.

27. Asztalos, B.F., et al. 2004. High-density lipoprotein subpopulation profile and coronary heart disease prevalence in male participants of the Framingham Offspring Study. Arterioscler. Thromb. Vasc. Biol. 24:2181-2187.

28. Freedman, D.S., et al. 2004. Sex and age differences in lipoprotein subclasses measured by nuclear magnetic resonance spectroscopy: the Framingham Study. Clin. Chem. 50:1189-1200.

29. Eriksson, M., Carlson, L.A., Miettinen, T.A., and Angelin, B. 1999. Stimulation of fecal steroid excretion after infusion of recombinant proapolipoprotein A-I. Potential reverse cholesterol transport in humans. Circulation. 100:594-598.

30. Parks, E.J., and Hellerstein, M.K. 2006. Thematic review series: patient-oriented research. Recent advances in liver triacylglycerol and fatty acid metabolism using stable isotope labeling techniques. J. Lipid Res. 47:1651-1660.

31. Ansell, B.J., Watson, K.E., Fogelman, A.M., Navab, M., and Fonarow, G.C. 2005. High-density lipoprotein function recent advances. J. Am. Coll. Cardiol. 46:1792-1798.

\title{
Autoantibody selection and production in early human life
}

\author{
Eric Meffre and Jane E. Salmon
}

Hospital for Special Surgery, Weill Medical College of Cornell University, New York, New York, USA.

\begin{abstract}
Natural antibodies are autoreactive/polyreactive antibodies believed to be secreted in the absence of xenoantigens. The origin and functional role of this limited and selective autoimmunity are not clear, nor is the specificity and range of autoantigens that drive the development of $B$ cells producing natural antibodies. In this issue of the JCI, Merbl et al. report that in utero, humans generate natural IgM and IgA antibodies that recognize a uniform set of autoantigens (see the related article beginning on page 712), some of which are associated with autoimmune diseases. The authors postulate that this "autoimmunity" at birth favors the emergence of autoimmune diseases in later life. We present a molecular basis for the limited and common repertoire of antibodies produced by fetal $B$ cells, which may be distinct from the abnormalities in $B$ cell development described in patients with autoimmune diseases.
\end{abstract}

$\mathrm{B}$ cell tolerance checkpoints remove many developing self-reactive $\mathrm{B}$ cells from the adult repertoire, yet the presence of certain autoreactive B cells in normal individuals is revealed by the identification of serum autoantibodies referred to as natural antibodies $(1,2)$. Most natural antibodies are low-affinity IgM autoantibodies that are

Nonstandard abbreviations used: BCR, B cell receptor; CDR3, complementarity-determining region 3; D, diversity; $\mathrm{H}$, heavy chain; IgH, Ig heavy chain; J, joining; NMHC-II, nonmuscle myosin heavy chain type II; TDT, terminal deoxynucleotidyl transferase; V, variable; XLA, $\mathrm{X}$-linked agammaglobulinemia.

Conflict of interest: The authors have declared that no conflict of interest exists.

Citation for this article: J. Clin. Invest. 117:598-601 (2007). doi:10.1172/JCI31578. often polyreactive and bind to a broad range of self antigens $(2,3)$. In mice, natural antibody-secreting $B$ cells belong to a specific B cell subpopulation called B-1 cells, which preferentially reside in the peritoneal cavity (4). The fact that these peripheral autoreactive B cells are positively selected by self antigens seems paradoxical to $B$ cell tolerance, but raises the possibility that autoantibodies may in some cases perform useful functions (5). In humans, the origin of natural antibodies and the autoantigens that they recognize remain to be characterized. In this issue of the JCI, Merbl et al. (6), using an antigen microarray, identified specific autoantigens recognized by natural IgM and, to a lesser extent, IgA antibodies produced during human fetal and neonatal life (6). In addition, they found that autoantigens recognized by serum IgMs from newborns were remarkably similar among individuals, suggesting that in the absence of xenoantigens, specific autoantigens may select and stimulate autoreactive B cells to produce self-reactive natural IgM antibodies in fetuses (6). In contrast, IgM and IgA antibodies from mothers showed an antigenic recognition pattern different from that of their newborns. Furthermore, IgM and IgA recognition patterns were extremely diverse among the mothers studied, reflecting the distinct immunological histories of each individual. However, encounters with some common pathogens, such as Gram-negative bacteria, stimulated the production of antiLPS antibodies in all mothers (6).

\section{Potential origins of common natural antibody reactivity at birth}

What might account for the recognition of the same autoantigens by different individuals during early human development? Antibodies are generated by random recombination of Ig variable $(\mathrm{V})$, diversity (D), and joining $(\mathrm{J})$ gene segments during early B cell development. Analysis of $B$ cells from fetuses and neonates showed that this was not the case in early life $(7,8)$. Rather, Ig gene segment usage in early life is biased toward specific genes, thereby lim- 